\title{
A scoping literature review of service-level barriers for access and engagement with mental health services for children and young people.
}

\author{
Joanna K. Anderson' (corresponding author) \\ Email: joanna.anderson@medschl.cam.ac.uk \\ Emma Howarth
}

Email: emma.howarth@medschl.cam.ac.uk

Maris Vainre

Email: maris.vainre@medschl.cam.ac.uk

Peter B. Jones

Email: pbj21@cam.ac.uk

Ayla Humphrey

Email: ayla.humphrey@cpft.nhs.uk

\section{Introduction}

The worldwide-pooled prevalence of mental disorders in children and adolescents is estimated at $13.4 \%$ [1]. In the UK one in ten children aged 5-16 years suffer from a diagnosable mental health $(\mathrm{MH})$ condition while many more are experiencing symptoms that, whilst not reaching the threshold of clinical disorder, are a source of distress for children, young people and their families [2]. Only $25 \%$ of children with clinically significant $\mathrm{MH}$ problems receive specialist care [3], while 43\% report no MH related service contact at all [4]. MH problems in childhood, unless treated, have a high level of persistence [5], with some conditions persevering through adolescence and into adulthood [6]. It is estimated that $25 \%$ of children with emotional disorders and $43 \%$ with conduct disorder still have the same condition three years later [5]. A half of lifetime mental illnesses start by the age of 15 and $74 \%$ by the age of 18 , increasing still further among those who use specialist mental health services by their mid-20's [7]. Failure to address MH problems early in life not only affects individuals' long-term functioning and wellbeing, but also produces significant societal costs resulting from increased healthcare usage,

unemployment and antisocial behaviours [8]. Snell et al. estimated yearly additional health, social care and educational costs associated with children psychiatric disorders in the UK at around f1.47bn [9].

Ample evidence suggest that service-level barriers are only one of the key factors hampering timely connection of children and young people experiencing $\mathrm{MH}$ difficulties with appropriate supportive services [10-14]. Service-level barriers for access and engagement have been highlighted by different groups of service users, including the most vulnerable ones, as well as providers, and commissioners. A recent systematic review of barriers encountered by young people seeking $\mathrm{MH}$ support identified lack of knowledge about available services, difficult access and stress associated with help-seeking process among key barriers to accessing $\mathrm{MH}$ services [12]. Brown et al [10] systematically reviewed studies of barriers for access to $\mathrm{MH}$ support encountered by young people from at-risk groups including ethnic and sexual minorities, culturally and linguistically diverse, homeless, substance users and youth residing in remote and rural areas. Apart from barriers specific to particular at-risk groups (e.g. language barrier, cultural norms, fear of being shamed), key barriers included lack of awareness of available services, system and access restrictions, long waiting times, and inflexibility of services [10]. A systematic review by Reardon et al [13] focusing on parents' perception of barriers for access and engagement with services for children and adolescent experiencing $\mathrm{MH}$ difficulties identified structural and systemic issues, and complicated help seeking process among key factors hampering timely access to services. A systematic review by Gondek et al [11] named limited resources, lack of information about available services, extensive and inflexible policies and regulations, and inflexible treatment provision major barriers for delivery of person-centred care in child and young people MH services. A systematic review focusing on barriers encountered by primary care practitioners managing children and adolescents $\mathrm{MH}$ problems [15] identified extensive waiting lists, financial restrictions and insufficient resources as key factors hampering their efforts to provide $\mathrm{MH}$ support in primary care settings.

In accordance with research evidence, the UK Department of Health recognised timely access to services and treatment as a major barrier for achieving parity of esteem for $\mathrm{MH}[16,17]$. Improving access to care is a key principles underpinning the ongoing redesign of Children and Adolescent Mental Health Services (CAMHS) [18]; by 2020 the UK government aims to have developed a comprehensive set of access and waiting times standards on a par with those seen in physical health service $[18,19]$. The recent report, Children and Young People's Mental Health Taskforce "Future in Mind" outlines key principles of CAMHS improvements [18] including improving timely access to services. Further guidance sets out strategies to operationalise these principles over the next five years through local level improvement initiatives guided by Local Transformation Plans [20]. These plans are developed by local Clinical 
Commissioning Groups, working closely with Health and Wellbeing Board Partners, and with strong input from children, young people and those who care for them [20].

In 2015 CLAHRC East of England conducted a consensus study with service users and the children and young people's workforce to identify priorities for the delivery of community based CAMHS in the region and to inform the development of Local Transformation Plans. One of the key themes that emerged was the need to enhance access to services and support. In order to translate key priorities into action plans leading to successful transformation of CAMHS, the process needs to be informed and guided by evidence. Although there are a number of recently published literature reviews focusing on barriers to access encountered by various user groups [10-14], commissioners, policy makers and service managers often lack resources to analyse and synthesise a large body of evidence themselves. As a result they are not always able to interpret recommendations and community opinions within a scientifically informed context, risking implementing them in ways which do not optimise access and may erode efficacy [21, 22]. In order to provide decision makers with a readily accessible evidence-base to guide local CAMHS transformation, a scoping literature review was undertaken to identify and aggregate evidence relating to key service development priorities highlighted in the consensus study. This paper reports results of a scoping literature review of barriers children, young people and families encounter when accessing and engaging with CAMHS, and effective strategies to overcome them.

\section{Materials and methods}

One of purposes for conducting a scoping review is to synthesise and disseminate research results to audiences that otherwise would not have time or resources to conduct a review themselves [23]. A scoping review is designed to provide an overview of literature on a particular topic and to highlight potential research gaps. It provides quantitative summary and qualitative thematic analysis of findings, and discusses implications for research and practice [24], however is not meant to be exhaustive, and does not assess the quality of evidence [23, 24].

The review described in this paper was guided by a methodological framework for conducting scoping studies proposed by Arksey and O'Malley [23], and further advanced by Levac et al [24]. The final search strategy (See Table 1) was determined after the initial data charting searches [25] and consultations with a subject librarian, and included the following: i) searching electronic bibliographic databases: MEDLINE, CINAHL, PsycINFO and Web of Science; ii) hand searching of relevant journals for potentially relevant studies and literature reviews; iii) forward citation search of the reference lists of primary studies included in the review, and the reference lists of relevant, previously published reviews. A three stage screening process, conducted independently by two researchers, included screening titles and abstracts to remove obviously irrelevant publications followed by screening abstracts of remaining publications, and finally full texts of potentially relevant papers for compliance with inclusion/exclusion criteria.

Table 1: Search strategy

\begin{tabular}{|c|c|}
\hline Barriers for access and engagement with CAMHS & Improving access and engagement with CAMHS \\
\hline $\begin{array}{l}\text { AB ((mental health N2 care) OR (mental N2 healthcare) OR (mental health } \\
\text { N2 service*) OR (CAMHS) OR TI ((mental health N2 care) OR (mental N2 } \\
\text { healthcare) OR (mental health N2 service*) OR (CAMHS) AND AB (child* } \\
\text { OR adolescen* OR teen* OR young people OR young person* OR minor* } \\
\text { OR youth* OR infan*) OR TI (child* OR adolescen* OR teen* OR young } \\
\text { people OR young person* OR minor* OR youth* OR infan*) AND AB } \\
\text { ((barrier* OR problem* OR delay*) N3 (access* OR referr* OR engage* OR } \\
\text { utili*)) OR TI ((barrier* OR problem* OR delay*) N3 (access* OR referr* OR } \\
\text { engage* OR utili*)) }\end{array}$ & $\begin{array}{l}\text { AB ((mental health N2 care) OR (mental N2 healthcare) OR (mental health } \\
\text { N2 service*) OR (CAMHS) OR TI ((mental health N2 care) OR (mental N2 } \\
\text { healthcare) OR (mental health N2 service*) OR (CAMHS) AND AB (child* } \\
\text { OR adolescen* OR teen* OR young people OR young person* OR minor* } \\
\text { OR youth* OR infan*) OR TI (child* OR adolescen* OR teen* OR young } \\
\text { people OR young person* OR minor* OR youth* OR infan*) AND AB ((wait* } \\
\text { N3 (time* OR list)) OR (access time*)) OR TI ((wait* N3 (time* OR list)) OR } \\
\text { (access time*)) AND AB (((improv* OR enhanc*) N3 (access* OR refer* OR } \\
\text { engag*))) OR TI (((improv* OR enhanc*) N3 (access* OR refer* OR } \\
\text { engag*))) }\end{array}$ \\
\hline
\end{tabular}

\subsection{Inclusion/exclusion criteria}

Due to time restrictions and lack of resources for translation services, only publications written in English were included. Since healthcare systems have changed significantly over the last two decades, and it was unlikely that currently delivered MH services were developed based on evidence or policy documents published more than 25 years ago, we included papers published after 1990. We excluded papers that are not empirical or evidence-based, however we included papers synthesising and discussing results of existing studies, or providing an overview of relevant policies and initiatives designed to improve access to services (discussion papers). The World Health Organization, Mental Health Atlas, (2005), indicates that although the majority of global burden of mental health disorders is in low to middle income countries, ninety percent of global mental health resources are located in high-income countries [26]. Furthermore, only $6 \%$ of the research on mental health have been published in indexed journals from these countries [27]. The mental health care and the research of this care in low income countries is demonstrably different to that in developed countries and for this reason we only included studies conducted in countries classified as developed economies or economies in transition [28]. 
1 Exclude if not written in English.

2 Exclude if published before January 1990.

3 Exclude if not empirical, not evidence based, not reviews of other studies or not a policy document/guideline (exclude commentaries, letters, book reviews).

4 Exclude if not directly or indirectly focused on mental health service users age 0-25 years (i.e. studies with parents/carers of mental health service users, service providers will be included).

5 Exclude in no focus on mental health or mental disorders. Exclude if symptoms are associated with non-mental health disorder (e.g. behavioural problems associated with ADS)

6 Exclude if is no focus beyond treatment type. The focus must include service delivery and be relevant to community care (regardless of persons or organization providing services, and severity or duration of mental health condition).

7 Exclude if services are not delivered in community settings

8 Exclude if describes children and adolescents mental health services in developing countries (according to World Economic Situation and Prospects 2015).

\subsection{Data extraction and reporting}

Data from included full texts were extracted into tables. A list of key themes was developed and publications were grouped in accordance with the main theme represented in each paper. As recommended by Levac et al [24] both a numerical summary of existing evidence and narrative description of findings in relation to the research questions was provided. To summarize available evidence, draw conclusions and make recommendations narrative synthesis of evidence using the framework proposed by Popay et al [29] was carried out.

\section{Findings}

\subsection{Descriptive numerical summary}

Our searches identified 3177 papers on service-level barriers for access to CAMHS and treatment engagement, and 47 papers were selected for final inclusion.

Table 3: Characteristics of included studies: Barriers for access to CAMHS and treatment engagement

\begin{tabular}{|c|c|c|}
\hline Criterion & Characteristic & Number of studies \\
\hline \multirow[t]{4}{*}{ Year } & $1990-2000$ & 3 \\
\hline & $2001-2005$ & 7 \\
\hline & $2006-2010$ & 18 \\
\hline & 2011-2016 & 19 \\
\hline \multirow[t]{4}{*}{ Country } & Australia & 9 \\
\hline & UK & 4 \\
\hline & USA & 29 \\
\hline & Canada & 5 \\
\hline \multirow[t]{6}{*}{ Study design/publication type } & Qualitative & 17 \\
\hline & Cross sectional & 16 \\
\hline & Mix methods & 5 \\
\hline & Audit & 5 \\
\hline & Experimental & 1 \\
\hline & Discussion paper & 3 \\
\hline \multirow[t]{5}{*}{ Participants/data source } & Children and young people & 14 \\
\hline & Parents & 16 \\
\hline & $\mathrm{MH}$ providers & 11 \\
\hline & MH support staff & 5 \\
\hline & Service outcome data & 8 \\
\hline
\end{tabular}




\begin{tabular}{|l|l|r|}
\hline \multirow{5}{*}{ Setting } & Rural & 17 \\
\cline { 2 - 3 } & Mixed & 13 \\
\cline { 2 - 3 } & Urban & \\
\cline { 2 - 3 } & Suburban & \\
\cline { 2 - 3 } & Not reported & \\
\cline { 2 - 3 } & Not applicable & \\
\hline
\end{tabular}

Barrier for access/engagement Availability of $\mathrm{MH}$ specialist services Criterion

\begin{tabular}{|c|c|c|c|}
\hline 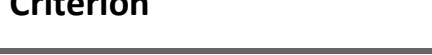 & \multicolumn{3}{|c|}{ Lack of information about available services } \\
\hline \multirow[t]{4}{*}{ Year } & $\begin{array}{l}\text { 1990-2000 } \\
\text { Inflexible services }\end{array}$ & 4 & \\
\hline & $\begin{array}{l}2001-2005 \\
\text { Waiting time }\end{array}$ & 4 & \\
\hline & $\begin{array}{l}\text { 2006-2010 } \\
\text { Complex administrative procedures }\end{array}$ & 8 & \\
\hline & $\begin{array}{l}\text { 2011-present } \\
\text { Costs associated with treatment }\end{array}$ & 19 & \\
\hline & \multicolumn{2}{|l|}{ Users' expectations of provider's attitude } & \\
\hline \multirow[t]{6}{*}{ Country } & \multicolumn{3}{|l|}{\begin{tabular}{l|l} 
USA & 7 \\
Barriers endorsed by primary care providers
\end{tabular}} \\
\hline & Canada & 3 & \\
\hline & Australia & 5 & \\
\hline & UK & 8 & \\
\hline & Finland & 1 & \\
\hline & New Zealand & 1 & \\
\hline \multirow[t]{7}{*}{ Study design } & Comparison group study & 5 & \\
\hline & RCT & 1 & \\
\hline & Audit & 7 & \\
\hline & Prospective cohort design & 1 & \\
\hline & Cross sectional design & 4 & \\
\hline & Mixed methods & 6 & \\
\hline & Qualitative (document analysis) & 1 & \\
\hline \multirow[t]{4}{*}{ Participants/data source } & Children and young people & 15 & \\
\hline & Parents & 2 & \\
\hline & MH service staff & 6 & \\
\hline & Records/service outcome data & 9 & \\
\hline \multirow[t]{5}{*}{ Setting } & Urban & 8 & \\
\hline & Mixed & 7 & \\
\hline & Suburban & 5 & \\
\hline & Rural & 1 & \\
\hline & Not reported & 5 & \\
\hline \multirow[t]{5}{*}{ Strategy to improve access } & Providing $\mathrm{MH}$ services in accessible location & 7 & \\
\hline & Facilitated access & 3 & \\
\hline & Case assessment and prioritisation & 7 & \\
\hline & Strategies to increase engagement & 5 & \\
\hline & Choice and Partnership Approach & 5 & \\
\hline
\end{tabular}

*Same papers may have reported more than one barrier

We identified 3859 papers on strategies to improve access and engagement, of which 25 were included in the review.

Table 4: Characteristics of included studies Improving access and engagement with CAMHS

20

11
11 10 14 18 10 Availability of specialist MH services

Twenty studies identified lack of availability of appropriate services as a major obstacle for connecting patients with $\mathrm{MH}$ difficulties with specialist services. Of those, seven were cross sectional studies using questionnaire, ten were qualitative studies involving interviews and focus groups with service users, one study used a mix of surveys and qualitative interviews, and one was a case study of service provision. Limited availability of specialised $\mathrm{MH}$ services was particularly cited by young people and families living in rural and remote areas. [30-34]. Traveling significant distance to see a $\mathrm{MH}$ specialist was a significant obstacle for parents $[31,35,36]$, while young people reported transportation problems as a barrier for accessing services [37-40]. Availability of services is not only a result of its geographical location, but also refers to access to $\mathrm{MH}$ support that is acceptable for users and available when needed. Two studies reported users' concerns about lack of after-hours and crisis support services [30,41], particularly when young people in crisis have to go through usual referral routes and face long wait lists to get help they need. Four studies cited lack of culturally appropriate $\mathrm{MH}$ services a barrier for access, especially for children and families from ethnic and racial minority groups [36, 42-45], while the same number reported language access barrier encountered by service users [44-47].

\section{Lack of information about available services}

Lack of information about available services resulting in

users not knowing where to go to get help was reported in eleven studies including three qualitative studies involving interviews and focus groups with service users, five cross sectional studies using questionnaire, and three case studies of service provision. Lack of information about available services was equally prominent in studies conducted in both rural and urban locations. Four studies reported that not knowing where service is located and how to make an appointment are major barriers for adolescents seeking $\mathrm{MH}$ support $[32,37,48,49]$. Not knowing where to go for MH support was also cited by a quarter out of 1948 participants of US Adolescent Health Care Access Survey [40]. One study reported that adolescents, even if they were aware of existing 
services, did not know how to make an appointment without approaching a school counsellor or a parent first [48]. Many of them did not want to involve an adult or did not attend school any longer, thus in these situations help-seeking was dependent on young person's prior knowledge of the service and the option of self-referral [48].

Parents also cited not knowing where to go as one of major barriers for seeking help for their children experiencing $\mathrm{MH}$ difficulties [50]. Results of a qualitative study examining issues for access to CAMHS in rural communities showed that in most cases families found out about available services through informal routes (i.e. from other people whose children were experiencing similar difficulties) [35].

\section{Inflexible services}

Nine studies reported inflexibility of services and lack of attempts to make it user-friendly as major obstacle for both initial access and continuous engagement with $\mathrm{MH}$ services. Of those, four were qualitative studies using interviews, three were cross sectional, survey based studies, one was a case study, and one study involved an audit of state mental health plans. Parents, especially when their child's treatment was ongoing, reported having difficulties with repeatedly taking time off work [50,51], and young people found it problematic that appointments were available only within school hours

[38]. One study reported that rigid rules according to which services operate caused difficulties in transferring records from one setting to another, resulting in treatment delays and disruption with taking medications due to inability to obtain prescription [52]. In another study school principals and counsellors reported that major obstacle for referring students with $\mathrm{MH}$ difficulties to specialist services is lack of flexibility of appointments and settings [41]

\section{Wait times}

Twenty one studies reported long wait times to be the major barrier for accessing and engaging with MH services. This included eight qualitative studies, six cross sectional studies, two studies using mixed methods, four audits of service data, and one case study. Long waiting times was the most commonly endorsed barriers cited by service users [37, 45, 48, 50, 53], healthcare professionals [54, 55] and service managers [56]. In a study by McCann et al [48] adolescents reported long wait time for an initial appointment a major obstacle for getting $\mathrm{MH}$ support. Due to high demand appointments had to be prioritised according to the level of urgency assessed by a clinician. Some adolescents expressed their frustration with the fact that their problems were not considered serious enough to get them seen soon, and this has deterred some from persevering with their appointment [48]. In two studies, users and parents expressed an opinion that the only way to 'jump the queue' was a child or young person doing something extreme, most commonly attempting suicide or self-harm, or threatening to harm others [30,35]. Over $60 \%$ out of 319 primary care providers reported that difficulty or delay in getting a $\mathrm{MH}$ specialist appointment was a major barrier for referring their adolescent patients to specialist services [55]. School principals and counsellors also identified long waiting lists as major obstacle for students' access to MH services, and indicated that long delays in accessing services often dissuaded them from making a referral to CAMHS [41].

Evidence suggest that long waiting lists have negative impact on families' engagement with $\mathrm{MH}$ services. There is a strong relationship between delay in scheduling an initial appointment, the length of time between referral and appointment date, and likelihood of attending the initial consultation [57-59]. Average wait time for kept appointments was 14.5 days and after 16.5 days the likelihood of keeping an appointment started to decrease with the odds of attending declining $1.4 \%$ for each additional day of wait time [60]. In a study by Schreader, almost a half of parents of children that were being placed on a waiting list contacted another agency. Longer waiting list increased the likelihood of seeking help elsewhere with $25 \%$ of families contacting another service within the first month of being on the waiting list [61].

\section{Complex administrative procedures}

Ten studies, including five qualitative studies, four survey based cross sectional studies and one audit of service data, reported administrative procedures and multiple referral steps major barriers for connecting children and young people with $\mathrm{MH}$ services. Parents indicated that having to make numerous phone calls to find a clinician, make appointments, reiterate the treatment history and explain the need for a visit is a significant barrier they encounter when seeking $\mathrm{MH}$ support for their child $[42,62]$. Having to deal with multiple referral steps to access the right service was another barrier reported by parents [50]. Service users often waited a long time to access one service only to be referred to another specialist and face another long waiting list [50]. A qualitative study examining experiences of parents seeking help for their child's MH difficulties found that parents, on average, contacted four agencies or professionals in the year preceding the study interview. Some parents were looking for help for different problems, however the majority contacted more than one agency in order to find the most appropriate treatment for the same problem [63]. In some cases, parents reported being offered a treatment they did not want for their child and declining it, to subsequently look for other options from different agencies and face another long wait for service [63]. Parents reported that overcoming administrative barriers for access to CAMHS requires advocacy work by a family member described as "being demanding, persisting, vocal, pushy, complaining, writing letters and going to the top" [35]. Parents acknowledged that they often did not get service because they did not push hard enough due to their personal style, lack of education and lack of time resulting from work obligations and parenting of other children [35].

\section{Costs associated with treatment}

Fourteen studies reported costs associated with treatment and insurance problems as significant barriers for seeking support for their children experiencing $\mathrm{MH}$ difficulties. Of these five were qualitative studies using interviews, seven questionnaire-based cross sectional studies, one audit of state mental health 
plans, and one case study. Since in the UK MH services for children are free under the National Health Service, this section reports findings from US and Australian studies. Included studies reported a range from $10 \%$ to $56 \%$ of respondents recounting costs associated with ongoing treatment, including costs of transport and organising care for other children, as a factor preventing them from engaging with services. One recent study reported that expenses associated with treatment were the highest ranked barrier endorsed by 52\% of 134 parents [50]. Respondents were not aware that child $\mathrm{MH}$ services in Australia did not charge any fees, indicating lack of knowledge how the support system works [50]. Another study evaluating Australian headspace showed that although this service for young people is initially free, Government's universal public health care system covers only 12 sessions per year, which means that some young people drop off mid-treatment, as they are not able to pay for the service [48]. A similar issue was highlighted by a US study of paediatricians who reported that children discontinue treatment prematurely due to limitations enforced by insurance programs [54]. Difficulties finding $\mathrm{MH}$ providers accepting Medicaid insurance was recognised as the major barrier for referring children with MH difficulties from primary care to specialist services [54]. In some US studies, parents reported that dealing with insurance companies impeded or delayed their attempts to access MH services [42-44, $53,54]$. For example with some insurance plans users need to obtain pre-authorisation for child's MH treatment from a health plan representative, which requires additional time and ability to navigate through a complicated system [42].

\section{Users' expectations of providers' attitudes}

Primary care providers are in most cases the first point of contact with services for parents concerned about their child's $\mathrm{MH}$. Eighteen studies reported that users' concerns about providers' attitude can be a major barrier to seeking and accessing MH support. These included six qualitative studies using interviews and focus groups, six cross sectional survey studies, three studies using mixed methods, two case studies and one audit of service outcomes data. Parents reported having concerns about $\mathrm{MH}$ professionals not caring about their children, being disrespectful, unfriendly, or committing the child against their will $[43,64]$. Some parents reported difficulties convincing a provider that their child was having a problem [42, 65]. Ambivalent or dismissive responses from a provider discouraged some parents from seeking help further, however some reported becoming more persistent [42]. In a study of 10,688 caregivers of children with special needs including $\mathrm{MH}$ difficulties, $31 \%$ of participants reported that lack of shared decision making discouraged them from continuing involvement with services [53]. According to users, some primary care providers offered medications before trying other, nonpharmaceutical approaches that are, in most cases, preferred by parents [42, 43] and adolescents [52, 66]. Parents who do not want to medicate their children without reviewing other options may seek another opinion, or ignore provider's recommendation and temporarily give up on seeking help until the child's problem becomes more severe [42]. Parents of adopted children cited $\mathrm{MH}$ professional not recognising the extent of parental challenge and feeling like they are being blamed for their adopted child's MH problems as barriers for seeking MH support [45]. Concerns about attitudes of healthcare professionals were also raised by young people experiencing $\mathrm{MH}$ difficulties. In two studies adolescents reported that concerns about not being treated with respect and worries about what a provider would say or do prevented them from seeking help [40,67]. In a study with adolescents diagnosed with depression and currently in treatment, participants who felt that their provider did not give them feedback or information, judged them as abnormal, or offered medication without discussing alternative options were less likely to comply with treatment recommendations [66]. Young people also expressed concerns about privacy and confidentiality, and the provider sharing what they revealed during a session with their parents [37, 40, 49, 67]. Discrepancies between users' and providers' perception of severity of $\mathrm{MH}$ difficulties reflected in cases prioritisation can also hamper patients' connection with $\mathrm{MH}$ services $[68,69]$. One study showed that the majority of youth who considered their MH difficulty severe were not referred to specialist services, as primary care physicians did not consider their problem serious enough to require specialist consultation [69].

\section{Service level barriers endorsed specifically by primary care providers}

Ten studies examined barriers to supporting children experiencing poor $\mathrm{MH}$ encountered by providers working in primary care settings. Six were questionnaire based cross sectional studies, three were qualitative studies involving interviews and focus groups, and one study used a mix of qualitative and quantitative methods. Paediatricians working in primary care settings reported that they do not feel sufficiently prepared to diagnose or treat $\mathrm{MH}$ problems [70]. However $97 \%$ out of 181 paediatricians felt comfortable prescribing stimulant medication, 59\% selective serotonin reuptake inhibitors, but only $25 \%$ antipsychotic medications [70]. One study showed that paediatricians and psychiatrists often disagreed as to the frequency with which particular $\mathrm{MH}$ problem should be referred. In cases of all disagreements, paediatricians indicated that the problems did not require specialist's involvement, while psychiatrists recommended referral [71].

Two studies cited lack of financial compensation for providing $\mathrm{MH}$ services and having to use physical health billing codes for behavioural health services as main reasons for not performing $\mathrm{MH}$ screening in primary care $[72,73]$. Two studies identified lack of MH professionals accessible for consultations and referrals as a major barrier [36, 70], and one study reported that unavailability of paediatric $\mathrm{MH}$ specialists resulted in pressure to refer adolescents to adult $\mathrm{MH}$ specialist [55]. In a study with 319 primary care clinicians, most commonly reported barriers for referring children and young people to specialist MH services included complex authorisation procedures, burdensome authorisation paperwork, difficulties finding MH specialist accepting particular insurance plan, financial disincentives and pressure to contain costs, and physicians' panel restrictions [55]. Three studies reported lack of communication between agencies and fragmentation of services as barriers for referring young people experiencing $\mathrm{MH}$ difficulties from primary care to specialist $\mathrm{MH}$ services [36, 70, 74]. Senior managers of children MH centres endorsed funding, case complexity, waitlists, staffing and lack of integration as major barriers for providing effective and timely care [56] 


\subsubsection{Strategies to improve access and engagement with $\mathrm{MH}$ services}

\section{Providing $\mathrm{MH}$ services at easily accessible locations}

Ten studies, including seven quantitative and three mixed methods studies, reported that providing MH services in easily accessible locations like schools, local primary care clinics or community walk-in clinics is an effective strategy to increase access and engagement, especially for users from hard to reach groups. In two studies MH services were provided weekly in schools by clinical psychologists and trainees from an outpatient, university affiliated MH clinic [75]. The first study found that children using school-based MH services did not differ in regard to clinical severity of MH problems from children seen in a central clinic, however, over a 12 months period, $98 \%$ of children attending school-based clinic were from minority population comparing to $37 \%$ in the central clinic [75]. In the second study all students $(n=1413)$ accessing school-based services during the first year of its activity were from ethnic minority groups [76]. A pilot study evaluating the impact of establishing a school MH consultation clinic, teachers' training and seminars for parents to increase their understanding of $\mathrm{MH}$ issues showed that the initiative improved access to $\mathrm{MH}$ services for young people who otherwise would have not sought help through traditional referral pathways [77]. Engaging parents and teachers in designing school MH services results in significantly increased family engagement with services and reduces treatment drop-out rates [78]

Evidence suggest that providing $\mathrm{MH}$ care in primary care settings increases the number of patients receiving appropriate and timely MH support. In a study examining the effectiveness of collaborative care model in which child psychiatrists offered real-time phone consultation and, if needed, further evaluation of patients seen in a paediatric clinic, about $40 \%$ of possible referrals were resolved during phone consultation with psychiatrist or psychiatric nurse [79]. Of all youths referred to specialist clinic, $46 \%$ were only seen once for an evaluation visit and afterwards they returned to primary care paediatrician for ongoing $\mathrm{MH}$ treatment. The program allowed psychiatrists to influence treatment of a large number of youths with relatively common and uncomplicated $\mathrm{MH}$ problems. Referring those cases back to primary care increased psychiatric clinic's capacity to provide ongoing care for patients with more complex problems, and reduce wait times [79]. An RCT evaluated effectiveness of an initiative that included placing an expert team leader at each of the five participating clinics, training care managers in manualised CBT for depression, and training clinicians in evaluation and treatment of depression [80] Patients from intervention sites had significantly higher rate of $\mathrm{MH}$ care and psychotherapy or counselling fewer depression symptoms, better quality of life, and higher satisfaction with care they received, comparing to patients in usual care [80].

One study showed that providing MH services to homeless in easily accessible location - an outreach clinic operating at youth crisis accommodation service - increased contact with services for those in need, and facilitated ongoing care with over a half of users attending more than one appointment [81]

\section{Facilitated access}

Results of three quantitative studies suggest that providing $\mathrm{MH}$ support in community walk-in clinics improves access to care for patients who are otherwise discouraged by complicated referral procedures and long waiting lists. Users attending a walk-in counselling centre found wait times more reasonable and had higher regard for counsellors' availability and service cultural sensitivity comparing to users in usual care [82]. Although walk-in clinic patients' had more severe $\mathrm{MH}$ problems comparing to those in usual care, they improved more quickly and required fewer appointments [82]. Some evidence suggest that self-referral is more acceptable way of accessing $\mathrm{MH}$ services for patients representing ethnic minority groups. A study evaluating acceptability of a self-referral MH service for Black and ethnic minority children and their families showed that this service model was more accessible for minority users comparing to usual care [83]. Similar results were found in a study evaluating self-referral MH service for Australian ethnic minority youth, however participating clinic also focused on providing culturally appropriate services, which could significantly contribute to its higher acceptability for this particular user group [84].

\section{Case assessment and prioritisation}

In seven quantitative studies, including three audits of service outcome data, assessing cases referred to $\mathrm{MH}$ specialist services and prioritising those that due to their severity require urgent attention was an effective strategy to reduce wait times for specialist appointments, and increase patients' flow. Two studies showed the effectiveness of a referral management system in which clinicians dedicated two days per month for evaluation appointments [85, 86]. Outcomes of these appointments were reviewed by a multidisciplinary team that decided cases' allocation and further management. Appointment slots were only given to families who confirmed their attendance. In both studies waiting times were significantly reduced [85, 86], and in one study the 'did not attend' rate decreased [85]. Another study evaluated a similar prioritisation model in which a multidisciplinary team provided up to five new referral slots per week [87]. New clients were offered up to three initial assessment appointments, followed by therapy or consultations with key agencies. Waiting time for initial appointment was reduced from 8 months to 8 weeks and a third of clients required only three sessions or fewer [87].

Standardised tools have been developed to facilitate case prioritisation using evidence-based criteria. Highland Prioritisation Criteria include six predicators of positive outcomes: having a single problem for less than 6 months, being under 5 years of age, changing schools or in exam year, being from a family with stable recent history of family composition and highly motivated to change [88]. All cases fulfilling at least four criteria were place on 'soon' waiting list, however families were required to confirm they want to wait, otherwise they were removed from the list. Introducing the criteria reduced wait time from 58 to 45 weeks and 'did not attend' rate dropped from 39\% to 13\% [88]. A Western Canada Waiting List (WCWL) developed and tested clinician-scored 
priority criteria measures, to objectively assess and compare the relative urgency of cases placed on waiting lists [89]. The initial version of WCWL prioritisation measure was developed by a panel including $\mathrm{MH}$ specialists, primary care physicians, and researchers. The criteria accounted for about $40 \%$ of observed variance in overall urgency ratings, and reliability assessment indicated half of the items had excellent or good interrater agreement, and testretest reliability [89]. Cawthorpe et al proposed a Child Mental Health Priority Criteria Score (CMH-PSC) based on initially developed WCWL measure [90]. The measure, completed in 2-4 minutes, has an excellent internal validity, significantly discriminates each clinical level, and its' score is meaningfully related to clinician's perceived urgency and maximum acceptable waiting time [90]. Kaukonen et al developed and tested a Finish nationwide standardised criteria for assessing the need for non-urgent child psychiatric care [91]. The Finish Child Psychiatric Criteria Tool, covering the entire case-mix of child psychiatric disorders, has $82 \%$ sensitivity and $74 \%$ specificity, with most items at least moderately correlated with clinical necessity-for-treatment assessment [91].

\section{Strategies to improve engagement}

Five studies focused on evaluating the effectiveness of strategies developed to reduce 'did not attend' rates. One study examined the impact of a learning collaborative that provided training to $\mathrm{MH}$ clinic staff on two evidence-based engagement strategies: a phone-based intervention and face-to-face interview [92]. Service outcome data were examined to compare attendance rates in 1-5 month periods in a year before and after introducing engagement strategies. Implementation of described strategies resulted in increasing initial intake from $5 \%$ to $21 \%$, while keeping subsequent appointments increased from $2 \%$ to $16 \%$ [92]. Evidence suggest that requiring patients to confirm their willingness to remain on a waiting list and to attend an initial appointment are effective strategies to reduce 'did not attend' rates $[85,86,88]$. However in one study only a quarter of families confirmed that they want to continue waiting for services. [93]. Of patients who were removed from the list 10\% were re-referred in 3-20 months and their time in treatment was on average 8 month longer comparing to those who opted to continue waiting [93]. In another study, letters with information about the length of the waiting list, and a child functional assessment questionnaire with a request to return to the clinic before or during the first appointment were sent to all referred patients [94]. A reminder call was made 1-2 days before the initial visit. There were no significant differences in the first appointment attendance rates between patients who did and did not receive the letter. However patients who returned the questionnaire prior to their visit were more likely to attend, comparing to those who did not [94].

\section{Choice and Partnership Approach (CAPA)}

CAPA approach has been widely adopted in the UK, Australia and New Zealand as a strategy to increase efficient use of limited MH service resources [9597]. CAPA is a patient management system informed by demand and capacity theory that emphasis patients' empowerment by putting them in charge of choosing a treatment they feel is most appropriate for them. Five studies (one qualitative study of service providers, two studies using mixed methods and one audit of service outcome data) examined the impact of implementing CAPA on waiting times, patient flow and users' and providers' satisfaction [95, 98]. In one study introducing CAPA virtually eliminated waiting lists and time between referral and first appointment decreased from 64 to 11 days [99]. However outcomes of CAPA evaluation involving six CAMHS teams across the UK suggest that it is beneficial and provides teams with well-structured planning model only when it is well-managed and implemented $[96,97]$. If it is implemented without appropriate management it may cause administrative problems, confusion and overworking of staff [97].

\section{Discussion and conclusions}

\subsection{Breadth of literature}

The review identified a number of service-level barriers for access and engagement with $\mathrm{MH}$ services encountered by children, young people and their families. Most studies that examined barriers for access focused on personal-level as well as service-level barriers providing a more comprehensive picture. Barriers were examined from perspectives of different stakeholders including service users (young people and their parent/carers), primary care and $\mathrm{MH}$ clinicians, and service managers. Most reported studies were conducted after 2010; before year 2005, studies examining barriers for access and engagement with $\mathrm{MH}$ care were scarce. This reflects the lag in demand and capacity in practice to emerge in research, as well as increasing interest in barriers for access encountered by ethnic minority groups. Over $60 \%$ of reported studies were conducted in the US and only four in the UK. The majority of reported studies are questionnaire-based cross-sectional studies and qualitative studies involving semi-structured interviews and focus groups with stakeholders. The literature on strategies to improve young people's access and engagement with MH services also emerged more recently. The majority of reviewed papers were published in the UK after year 2010, and examine strategies to decrease waiting times for $\mathrm{MH}$ services. Audits of service outcome data and administrative case files were most frequent, followed by questionnaire-based cross sectional and comparison group studies.

\subsection{Findings}

One of the major barriers for seeking help with $\mathrm{MH}$ problems reported by both young people and their parents/carers is lack of awareness of existing $\mathrm{MH}$ services, and limited understanding of pathways to access appropriate care [31, 35, 37, 39, 40,43]. This may be due to the fact that although services are available for young people and their families, they are not particularly well publicised, and potential users have to 'ask around' to find out where to seek help. Most specialised services cannot be accessed directly and referral routes are complex and hard to navigate, especially for users who are not very well equipped to negotiate the system, e.g. due to a language barrier [47] or personal style preventing parents from advocating strongly to access services they 
think are optimal for their child [42]. Connecting with appropriate services is often a drawn-out, time consuming process, which for parents who have work and childcare obligations can be a major barrier for seeking help [42, 50,62]. Making MH services more visible for potential users through online presence or advertising in places often visited by young people, as well as providing step-by-step help-seeking guidelines explaining in a user-friendly manner the process of connecting with services may facilitate access to $\mathrm{MH}$ care. Also providing advocacy support for service users, especially more vulnerable ones who may find navigating complicated referral system particularly difficult, seems to be effective strategy to improve access to $\mathrm{MH}$ care [35]. Being able to get specialist appointment only during parents' working hour and children's school hours can be a major barrier for engagement with MH service, especially if young person's treatment is ongoing $[38,50]$. Designing services around users' needs and preferences can potentially increase uptake and continuous engagement with service [38]. Limited availability and distant geographical location of specialist MH services are barriers for access especially prominent in rural and remote areas [37, 48]. Evidence strongly suggests that using technology to deliver MH services (e.g. video-conferencing) can be an effective way of increasing access for users who are not in a proximity to a specialist $\mathrm{MH}$ clinic [100]. Young people experiencing MH difficulties may not want to nor be able to involve an adult in help-seeking process $[40,48]$. They are often concerned about the attitude of primary care provider they have to approach first to access specialist services [40]. One way of solving this is to enable young people to directly access MH services through self-referral, and ensuring that young people are aware of this access route. This strategy proved to effectively facilitate access for young people who were unlikely to connect with services through traditional access routes [75-78, 81, 82, 84]. Providing assessment and treatment of common MH disorders in primary care settings improves patients' access to $\mathrm{MH}$ care and contributes to reducing waiting times and pressure on specialised services, increasing their capacity to support patients with more complex $\mathrm{MH}$ needs [79, 80]. However, evidence suggests that primary care clinicians may not have sufficient knowledge to provide $\mathrm{MH}$ support [70] and have to deal with numerous administrative barriers when they try to connect families with specialised services [36, 55, 72-74]. Training and supporting primary care clinicians to offer initial assessment and treatment for common $\mathrm{MH}$ problems, and enhancing communication between primary care and children psychiatric services might improve young people's timely access to MH support. One of the most prominent barriers for seeking help with $\mathrm{MH}$ problems reported by a range of stakeholders is long waiting time to receive specialist care [37, 45, 48, 50, 53-56]. A number of strategies have been proposed to tackle the issue of long waiting lists, however none provides an ideal solution. Case assessment and prioritization by multidisciplinary teams based on clinical urgency often does not reflect clinicians', parents' and young persons' perception of seriousness of their problem $[68,69]$. This discrepancy may, in crisis situations, drive young people to take extreme actions (i.e. attempted suicide or self-harm) to ensure quick access to services [30, 35]. Prioritization based on standardised urgency assessment measures with proven psychometric properties can be a good alternative, ensuring more objective, evidence-based allocation of cases [89-91]. Another common strategy to reduce waiting times is requiring families to confirm their willingness to remain on a waiting list or otherwise they are not offered services $[85,86,88]$. Some evidence suggest that not only do a small number of families confirm they want to continue waiting for services, but also those who are removed from the waiting list are likely to be re-referred with more severe disorder [98]. MH problems in childhood tend to persevere if not appropriately addressed [5], thus removing patients from a waiting list and delaying their connection with services is likely to have detrimental effect on their MH. Evidence suggests that engaging with families placed on a waiting list rather than just requiring them to confirm their intention to continue waiting for services is an effective strategy to increase an uptake of initial appointment and subsequent engagement with services. Brief interventions provided by intake workers are time and cost-effective and can significantly improve attendance, and thus contribute to shortening waiting times [92, 94].

\subsection{Research gaps}

This review identified prominent research gaps, mainly regarding methodology of conducted studies. The majority of studies examining barriers for young people's access to MH services include small samples affecting generalisability of findings. Most studies involve convenience samples of participants who have already been in contact with services for some time. Very few studies examine perspectives of young people who are considering seeking MH help for the first time $[37,40,49,53]$, but expect to encounter particular barriers that would impede access to appropriate services. Nearly $30 \%$ of studies evaluating strategies to improve access to $\mathrm{MH}$ services are audits of available service outcome data. They usually encompass relatively short period of time prior and after introducing an intervention (12 months at the most), thus there is no evidence on how effective particular approaches are over a longer period of time. Additionally most studies only examine the impact of strategies to improve access on waiting times and patient flow. It is possible that in a long run those strategies would have impact on clinical outcomes, patients' satisfaction, clinical and administrative staff's workload, job satisfaction and costs of providing services, but at the moment available evidence does not permit drawing conclusions about long-term impact. Controlled trials with longer term follow-up are needed to establish the long-term effectives of access improving strategies, and their impact on patient, provider and service levels.

\subsection{Limitation}

This scoping review has a pragmatic aim to provide a readily available evidence-base to support development and implementation of CAMHS Local Transformation Plan [20] in the East of England. It was designed to enable policy makers, commissioners and service managers to make decisions about redesigning, implementing and evaluating CAMHS within scientifically supported context, to ensure optimal outcomes. As a result, the review focuses largely on barriers for access associated with the way $\mathrm{MH}$ services for children and adolescents are designed, and pragmatic strategies to overcome them, 
therefore it may not provide a complete overview of existing evidence. Having in mind the pragmatic purpose of the review, we excluded studies where results were not, in our opinion, applicable in the UK context, mainly due to differences in the way CAMHS are designed and delivered in different countries, or populations they serve. Although mental health care systems and populations differ between the countries, this does not detract from the significance of the commonalties which we discuss. We have clearly indicated where there are issues relevant to particular countries, e.g. in payment structures. A comprehensive scoping of the literature is bound to be weighted towards the US where there is a long history of research in this area. The findings, however, clearly speak to the situation in the UK, for example, and replicate concerns raised in "Future in Mind" as well as the recent Delphi study examining priorities for CAMHS provision (Howard et al., in preparation). We excluded publications that did not focus solely on $\mathrm{MH}$ conditions, i.e. we excluded a large number of papers describing barriers to access to $\mathrm{MH}$ services for users with developmental or learning disabilities, or whose $\mathrm{MH}$ symptoms did not explicitly result from a MH disorder (e.g. behavioural problems in children with ADHD). Finally, since we have not assessed the quality of included evidence, the review comprises a number of studies that are not particularly strong methodologically thus their results, however promising, have to be interpreted with great caution. The review includes a number of case studies examining implementation of a particular service model or strategy to improve access and engagement with $\mathrm{MH}$ services. Some of these strategies produced very promising outcomes, however they were implemented and evaluated in small, homogenous organisations and over a short period of time. As a result it is hard to make predictions whether the same intervention would produce equally positive outcome if implemented in different settings and evaluated over a longer period of time. Many reported studies included very small sample of self-selected participants, increasing likelihood of biased results. Due to quality of available evidence, outcomes of this review have to be interpreted with caution. Their gravity is not sufficient to support definitive conclusions regarding optimal strategies to address most prominent servicelevel barriers that prevent children and young people from accessing and engaging with $\mathrm{MH}$ services.

We confirm that there are no known conflicts of interest associated with this publication. We confirm that the manuscript has been read and approved by all named authors and that there are no other persons who satisfied the criteria for authorship but are not listed. We further confirm that the order of authors listed in the manuscript has been approved by all of us.

\section{References:}

1. Polanczyk, G.V., Salum, G. A., Sugaya, L. S., Caye, A., \& Rohde, L. A. , Annual Research Review: A meta - analysis of the worldwide prevalence of mental disorders in children and adolescents. . Journal of Child Psychology and Psychiatry, 2015. 56(3): p. $345-365$.

2. Green, H., et al., Mental health of children and young people in Great Britain, 2004. 2005.

3. Hagell, A., J. Coleman, and F. Brooks, Key Data on Adolescence 2015. 2015, Association for Young People's Health: London

4. Ford, T., et al., Service contacts among the children participating in the British Child and Adolescent Mental Health Surveys. Child \& Adolescent Mental Health, 2005. 10(1): p. 2-9 8p.

5. Meltzer, H., et al., Persistence, onset, risk factors and outcomes of childhood mental disorders. 2003, TSO: London.

6. Woodward, L.J. and D.M. Fergusson, Life course outcomes of young people with anxiety disorders in adolescence. Journal of the American Academy of Child \& Adolescent Psychiatry, 2001. 40(9): p. 1086-1093.

7. Kim-Cohen, J., Caspi, A., Moffitt, T. E., Harrington, H., Milne, B. J., \& Kim-Cohen, J., Caspi, A., Moffitt, T.E., Harrington, H., Milne, B.J. and Poulton, R. Prior juvenile diagnoses in adults with mental disorder: developmental follow-back of a prospective-longitudinal cohort. . Archives of general psychiatry, 2003. 60(7): p. 709-717.

8. Joint Commissioning Panel for Mental Health. Guidance for commissioners of children and adolescent mental health services. 2013.

9. Snell, T., et al., Economic impact of childhood psychiatric disorder on public sector services in Britain: estimates from national survey data. Journal of child psychology and psychiatry, 2013. 54(9): p. 977-985

10. Brown, A., et al., Systematic review of barriers and facilitators to accessing and engaging with mental health care among at-risk young people. AsiaPacific Psychiatry: Official Journal Of The Pacific Rim College Of Psychiatrists, 2015.

11. Gondek, D., Edbrooke - Childs, J., Velikonja, T., Chapman, L., Saunders, F., Hayes, D., \& Wolpert, M. , Facilitators and Barriers to Person - centred Care in Child and Young People Mental Health Services: A Systematic Review. Clinical Psychology \& Psychotherapy., 2016.

12. Gulliver, A., K.M. Griffiths, and H. Christensen, Perceived barriers and facilitators to mental health help-seeking in young people: A systematic review. BMC Psychiatry, 2010. 10.

13. Reardon, T., Harvey, K., Baranowska, M., O'Brien, D., Smith, L., \& Creswell, C. , What do parents perceive are the barriers and facilitators to accessing psychological treatment for mental health problems in children and adolescents? A systematic review of qualitative and quantitative studies. European Child \& Adolescent Psychiatry, 2016: p. 1-25.

14. Rowe, S.L., et al., Help-seeking behaviour and adolescent self-harm: a systematic review. The Australian And New Zealand Journal Of Psychiatry, 2014. 48(12): p. 1083-1095

15. O'Brien, D., Harvey, K., Howse, J., Reardon, T., \& Creswell, C. , Barriers to managing child and adolescent mental health problems: a systematic review of primary care practitioners' perceptions. Br J Gen Pract, 2016. bjgpoct-2016.

16. Department of Health, No health without mental health. A cross-government mental health outcomes strategy for people of all ages. 2011

17. Department of Health, Closing the gap: priorities for essential change in mental health. . 2014

18. Department of Health, Future in mind: promoting, protecting and improving our children and young people's mental health and wellbeing. 2015, Department of Health.

19. Department of Health, Achieving better access to mental health services by 2020. 2014.

20. NHS England, Transformation Plans for Children and young People's Mental Health and Wellbeing: Guidance and support for local areas. 2015.

21. Wye, L., Brangan, E., Cameron, A., Gabbay, J., Klein, J. H., \& Pope, C. , Evidence based policy making and the 'art'of commissioning-how English healthcare commissioners access and use information and academic research in 'real life'decision-making: an empirical qualitative study. . BMC health services research, 2015. 15(1): p. 430.

22. Clarke, A., Taylor-Phillips, S., Swan, J., Gkeredakis, E., Mills, P., Powell, J., ... \& Grove, A. , Evidence-based commissioning in the English NHS: who uses which sources of evidence? A survey 2010/2011. BMJ open,, 2013. 3(5): p. e002714.

23. Arksey, H. and L. O'Malley, Scoping studies: towards a methodological framework. International Journal of Social Research Methodology, 2005. 8(1): p. 19-32. 
25. Joanna Briggs Institute. Joanna Briggs Institute Reviewers' Manual 2015 edition, Methodology for JBI scoping reviews. 2015.

26. World Health Organization, W.P.A., International Association for Child, Adolescent Psychiatry, \& Allied Professions, , , Atlas: child and adolescent mental health resources: global concerns, implications for the future. 2005.

27. Saxena, S., Paraje, G., Sharan, P., Karam, G., Sadana, R. , The 10/90 divide in mental health research: trends over a 10-year period. The British Journal of Psychiatry, 2006. 188(1): p. 81-82.

28. United Nations. World Economic Situation and Prospects 2017. 2017.

29. Popay J, R.H., Sowden A, Petticrew M, Arai L, Rodgers M, Britten N, Roen K, Duffy S., Guidance on the conduct of narrative synthesis in systematic reviews. A product from the ESRC methods programme, 2006. Version 1: p. b92.

30. Aisbett, D.L., et al., Understanding barriers to mental health service utilization for adolescents in rural Australia. Rural \& Remote Health, 2007. 7(1): p. 624-624

31. Bringewatt, E.H. and E.T. Gershoff, Falling through the cracks: Gaps and barriers in the mental health system for America's disadvantaged children. Children and Youth Services Review, 2010. 32(10): p. 1291-1299.

32. Boyd, C., et al., Preferences and intention of rural adolescents toward seeking help for mental health problems. Rural \& Remote Health, 2011. 11(1): p. 1-13 13p.

33. Pullmann, M.D., et al., Barriers to and supports of family participation in a rural system of care for children with serious emotional problems. Community Mental Health Journal, 2010. 46(3): p. 211-220.

34. Boyd, C.P., et al., Issues in rural adolescent mental health in Australia. Rural And Remote Health, 2006. 6(1): p. $501-501$.

35. Boydell, K.M., et al., Family perspectives on pathways to mental health care for children and youth in rural communities. Journal of Rural Health, 2006. 22(2): p. 182-188 7p.

36. Bitar, G.W., et al., Barriers and facilitators of adolescent behavioral health in primary care: Perceptions of primary care providers. Families, Systems, \& Health, 2009. 27(4): p. 346-361.

37. Hernan, A., et al., Healthy minds for country youth: help-seeking for depression among rural adolescents. The Australian Journal Of Rural Health, 2010. 18(3): p. 118-124.

38. Muir, K., A. Powell, and S. McDermott, 'They don't treat you like a virus' : Youth - friendly lessons from the Australian national youth mental health foundation. Health \& Social Care in the Community, 2012. 20(2): p. 181-189.

39. Sakai, C., et al., Mental Health Beliefs and Barriers to Accessing Mental Health Services in Youth Aging out of Foster Care. Academic Pediatrics, 2014. 14(6): p. 565-573 9p.

40. Samargia, L.A., E.M. Saewyc, and B.A. Elliott, Foregone Mental Health Care and Self-Reported Access Barriers Among Adolescents. The Journal of School Nursing, 2006. 22(1): p. 17-24.

41. Allison, S., L. Roeger, and D. Abbot, Overcoming barriers in referral from schools to mental health services. Australasian Psychiatry, 2008. 16(1): $\mathrm{p}$. 44-47.

42. Cohen, E., et al., Parents' Perspectives on Access to Child and Adolescent Mental Health Services. Social Work in Mental Health, 2012. 10(4): p. 294$31017 \mathrm{p}$.

43. Richardson, L.A., Seeking and obtaining mental health services: what do parents expect? Archives Of Psychiatric Nursing, $2001.15(5)$ : p. 223-231.

44. Gould, S.R., S.E. Beals-Erickson, and M.C. Roberts, Gaps and barriers in services for children in state mental health plans. Journal of Child and Family Studies, 2012. 21(5): p. 767-774.

45. Golding, K.S., Multi-agency and specialist working to meet the mental health needs of children in care and adopted. Clinical Child Psychology and Psychiatry, 2010. 15(4): p. 573-587.

46. Cohen, P. and C.S. Hesselbart, Demographic factors in the use of children's mental health services. American Journal of Public Health, 1993. 83(1): p. 49-52 4p.

47. Williams, M.E., et al., Barriers to accessing services for young children. Journal of Early Intervention, 2013. 35(1): p. 61-74.

48. McCann, T.V. and D.I. Lubman, Young people with depression and their experience accessing an enhanced primary care service for youth with emerging mental health problems: A qualitative study. BMC Psychiatry, 2012. 12.

49. Boyd, C., et al., Australian rural adolescents' experiences of accessing psychological help for a mental health problem. Australian Journal of Rural Health, 2007. 15(3): p. 196-200 5p.

50. Iskra, W., et al., Parental perceptions of barriers to mental health services for young people. Early Intervention In Psychiatry, 2015.

51. DeRigne, L., What are the parent-reported reasons for unmet mental health needs in children? Health \& Social Work, 2010. 35(1): p. 3-15 13p.

52. Oruche, U.M., et al., Barriers and facilitators to treatment participation by adolescents in a community mental health clinic. Journal of Psychiatric and Mental Health Nursing, 2014. 21(3): p. 241-248.

53. Vohra, R., et al., Access to services, quality of care, and family impact for children with autism, other developmental disabilities, and other mental health conditions. Autism: The International Journal Of Research And Practice, 2014. 18(7): p. 815-826.

54. Pfefferle, S.G., Pediatrician perspectives on children's access to mental health services: Consequences and potential solutions. Administration and Policy in Mental Health and Mental Health Services Research, 2007. 34(5): p. 425-434.

55. Walders, N., et al., Barriers to mental health referral from pediatric primary care settings. The American Journal Of Managed Care, 2003. 9(10): $p$. 677-683.

56. Reid, G.J. and J.B. Brown, Money, case complexity, and wait lists: Perspectives on problems and solutions at children's mental health centers in Ontario. The Journal of Behavioral Health Services \& Research, 2008. 35(3): p. 334-346.

57. Stern, G. and R. Brown, The effect of a waiting list on attendance at initial appointments in a child and family clinic. Child: Care, Health and Development, 1994. 20(4): p. 219-230.

58. Westin, A., C. Barksdale, and S. Stephan, The Effect of Waiting Time on Youth Engagement to Evidence Based Treatments. Community Mental Health Journal, 2014. 50(2): p. 221-228 8p.

59. Gallucci, G., W. Swartz, and F. Hackerman, Impact of the Wait for an Initial Appointment on the Rate of Kept Appointments at a Mental Health Center. Psychiatric Services, 2005. 56(3): p. 344-346.

60. Sherman, M.L., et al., Clinical intake of child and adolescent consumers in a rural community mental health center: does wait-time predict attendance? Community Mental Health Journal, 2009. 45(1): p. 78-84 7p.

61. Schraeder, K.E. and G.J. Reid, Why wait? The effect of wait-times on subsequent help-seeking among families looking for children's mental health services. Journal of Abnormal Child Psychology, 2015. 43(3): p. 553-565.

62. Radovic, A., et al., Primary care providers' beliefs about teen and parent barriers to depression care. Journal of Developmental and Behavioral Pediatrics, 2014. 35(8): p. 534-538.

63. Reid, G.J., et al., Help-seeking for children with mental health problems: parents' efforts and experiences. Administration And Policy In Mental Health, 2011. 38(5): p. 384-397.

64. Flisher, A.J., et al., Correlates of unmet need for mental health services by children and adolescents. Psychological Medicine, 1997. 27(5): p. 11451154.

65. Brown, C.M., et al., Pediatricians may address barriers inadequately when referring low-income preschool-aged children to behavioral health services. Journal of Health Care for the Poor and Underserved, 2014. 25(1): p. 406-424. 
Wisdom, J.P., G.N. Clarke, and C.A. Green, What Teens Want: Barriers to Seeking Care for Depression. Administration and Policy in Mental Health and Mental Health Services Research, 2006. 33(2): p. 133-145.

67. Draucker, C.B., Processes of mental health service use by adolescents with depression. Journal Of Nursing Scholarship: An Official Publication Of Sigma Theta Tau International Honor Society Of Nursing / Sigma Theta Tau, 2005. 37(2): p. 155-162.

68. Maguire, S. and J. Guishard-Pine, Parents' and clinicians' perception of severity of referral problems. Child and Adolescent Mental Health, 2005. 10(4): p. 183-189.

69. Stiffman, A.R., et al., Youths' access to mental health services: the role of providers' training, resource connectivity, and assessment of need. Mental Health Services Research, 2000. 2(3): p. 141-154.

70. Davis, D.W., et al., Identification and management of behavioral/mental health problems in primary care pediatrics: perceived strengths, challenges, and new delivery models. Clinical Pediatrics, 2012. 51(10): p. 978-982.

71. Oke, S. and R. Mayer, Referrals to child psychiatry--a survey of staff attitudes. Archives Of Disease In Childhood, $1991.66(7)$ : p. 862-865.

72. Diamond, G.S., et al., Attitudes, practices, and barriers to adolescent suicide and mental health screening: a survey of pennsylvania primary care providers. Journal Of Primary Care \& Community Health, 2012. 3(1): p. 29-35.

73. Honigfeld, L. and K. McKay, Barriers to enhancing practice-based developmental services. Journal of Developmental and Behavioral Pediatrics, 2006. 27(Suppl1): p. S30-S33.

74. Macdonald, E., et al., Current delivery of infant mental health services: Are infant mental health needs being met? Australasian Psychiatry, 2005. 13(4): p. 393-398.

75. Armbruster, P., S.H. Gerstein, and T. Fallon, Bridging the gap between service need and service utilization: a school-based mental health program. Community Mental Health Journal, 1997. 33(3): p. 199-211.

76. Bear, L., et al., Building the gateway to success: An appraisal of progress in reaching underserved families and reducing racial disparities in schoolbased mental health. Psychological Services, 2014. 11(4): p. 388-397.

77. Eapen, V. and R. Jairam, Integration of child mental health services to primary care: challenges and opportunities. Mental Health in Family Medicine, 2009. 6(1): p. 43-48 6p.

78. Atkins, M.S., et al., Toward A New Model for Promoting Urban Children's Mental Health: Accessible, Effective, and Sustainable School-Based Mental Health Services. School Psychology Review, 2003. 32(4): p. 503-514.

79. Aupont, O., et al., A collaborative care model to improve access to pediatric mental health services. Administration and Policy in Mental Health and Mental Health Services Research, 2013. 40(4): p. 264-273.

80. Asarnow, J.R., et al., Effectiveness of a Quality Improvement Intervention for Adolescent Depression in Primary Care Clinics: A Randomized Controlled Trial. JAMA: Journal of the American Medical Association, 2005. 293(3): p. 311-319.

81. Dixon, C., et al., Linking young homeless people to mental health services: An exploration of an outreach clinic at a supported youth accommodation service. Advances in Mental Health, 2011. 10(1): p. 83-91.

82. Barwick, M., et al., Profiles and service utilization for children accessing a mental health walk-in clinic versus usual care. Journal of Evidence-Based Social Work, 2013. 10(4): p. 338-352.

83. Fatimilehin, I.A., Building bridges in Liverpool: delivering CAMHS to black and minority ethnic children and their families. Journal of Integrated Care, 2007. 15(3): p. 7-16 10p.

84. Clark, T.C., et al., Facilitating Access to Effective and Appropriate Care for Youth With Mild to Moderate Mental Health Concerns in New Zealand. Journal of Child \& Adolescent Psychiatric Nursing, 2014. 27(4): p. 190-200 11p.

85. Clemente, C., et al., Evaluation of a Waiting List Initiative in a Child and Adolescent Mental Health Service. Child and Adolescent Mental Health, 2006. 11(2): p. 98-103.

86. Jones, E., C. Lucey, and L. Wadland, Triage: A waiting list initiative in a child mental health service. Psychiatric Bulletin, 2000. 24(2): p. 57-59.

87. York, A., Y. Anderson, and M. Zwi, Eight months to eight weeks: reducing waiting times in a child and adolescent mental health service. Mental Health Review, 2004. 9(2): p. 15-19 5p.

88. Woodhouse, A.E., Reducing Waiting Times: Using an Opt-In System and Changing Prioritisation Criteria. Child and Adolescent Mental Health, 2006. 11(2): p. 94-97.

89. Smith, D.H. and D.C. Hadorn, Lining up for children's mental health services: A tool for prioritizing waiting lists. Journal of the American Academy of Child \& Adolescent Psychiatry, 2002. 41(4): p. 367-376.

90. Cawthorpe, D., et al., Priority-setting for children's mental health: Clinical usefulness and validity of the priority criteria score. Journal of the Canadian Academy of Child and Adolescent Psychiatry / Journal de l'Académie canadienne de psychiatrie de l'enfant et de l'adolescent, 2007. 16(1): p. 18-26.

91. Kaukonen, P., et al., Child psychiatry in the Finnish health care reform: National criteria for treatment access. Health Policy, 2010. 96(1): p. 20-27.

92. Cavaleri, M.A., et al., The effect of a learning collaborative to improve engagement in child mental health services. Children and Youth Services Review, 2010. 32(2): p. 281-285.

93. Walker, S., Mental health. Wait not, want not? The Health Service Journal, 1998. 108(5607): p. 32-33.

94. Parker, K.C. and A.P. Froese, Waiting list information strategies for child psychiatry: an intervention and measurement approach. Canadian Journal Of Psychiatry. Revue Canadienne De Psychiatrie, 1992. 37(6): p. 387-392.

95. Fuggle, P., et al., Can we improve service efficiency in CAMHS using the CAPA approach without reducing treatment effectiveness? Journal Of Child Health Care: For Professionals Working With Children In The Hospital And Community, 2015.

96. Robotham, D., Evaluation of the Choice and Partnership Approach (CAPA) in child and adolescent mental health services in England. 2009, Mental Health Foundation.

97. Robotham, D.J., K., Cyhlarova, E. , Managing demand and capacity within child and adolescent mental health services: an evaluation of the Choice and Partnership Approach. Mental Health Review Journal, 2010. 13(3): p. 22-30.

98. Wilson, S.M., J., \& McLeod, S., Comparing choice and partnership approach assumptions to child and adolescent mental health services in NHS Greater Glasgow and Clyde. International journal of health care quality assurance, 2015. 28(8): p. 812-825.

99. Naughton, J., et al., Improving quality of a rural CAMHS service using the Choice and Partnership Approach. Australasian Psychiatry, 2015. 23(5): p. 561-565.

100. Boydell, K.M., et al., Using technology to deliver mental health services to children and youth: a scoping review. . Journal of the Canadian Academy of Child \& Adolescent Psychiatry, 2014. 23(2). 
Table 5: Findings - barriers for access to CAMHS and treatment engagement

\begin{tabular}{|c|c|c|c|c|c|c|c|c|c|c|c|c|c|c|c|c|c|c|}
\hline Reference & Year & Country & $\begin{array}{l}\begin{array}{l}\text { Study } \\
\text { design }\end{array} \\
\end{array}$ & CYP & Parents & $\begin{array}{l}\mathrm{MH} \\
\text { providers }\end{array}$ & $\begin{array}{l}\text { MH support } \\
\text { staff }\end{array}$ & $\begin{array}{l}\text { Service outcome } \\
\text { data }\end{array}$ & $\begin{array}{l}\text { Sample } \\
\text { size }\end{array}$ & Setting & $\begin{array}{l}\text { Barriers in } \\
\text { PC }\end{array}$ & $\begin{array}{l}\text { Service } \\
\text { availability }\end{array}$ & $\begin{array}{l}\text { Lack of } \\
\text { information }\end{array}$ & $\begin{array}{l}\text { Inflexible } \\
\text { services }\end{array}$ & $\begin{array}{l}\text { Waiting } \\
\text { time }\end{array}$ & $\begin{array}{l}\text { Administrative } \\
\text { procedures }\end{array}$ & cost & $\begin{array}{l}\text { Provider's } \\
\text { attitude }\end{array}$ \\
\hline Aisbett et al & 2007 & Australia & Qual & $x$ & & & & & 3 & Rural & & $\mathrm{x}$ & & $\mathrm{x}$ & $\mathrm{x}$ & & & \\
\hline Allison et al & 2008 & Australia & CS & & & & $\mathrm{x}$ & & 171 & Mixed & & $x$ & & $x$ & $x$ & & & \\
\hline Allnock et al & 2012 & UK & MM & $\mathrm{x}$ & & & $x$ & $x$ & 269 & Mixed & $x$ & $x$ & & & $x$ & & & \\
\hline Bitar et al & 2009 & USA & Qual & & & $x$ & & & 181 & Mixed & $x$ & $x$ & & $x$ & & & $x$ & \\
\hline Boyd et al & 2007 & Australia & CAS & & & & & & NA & Rural & & $x$ & $x$ & & & & $x$ & $x$ \\
\hline Boyd et al & 2011 & Australia & cs & $\mathrm{x}$ & & & & & 201 & Rural & & $x$ & & & & & $x$ & \\
\hline Boydell et al & 2006 & Canada & Qual & & $x$ & & & & 30 & Rural & & $x$ & $x$ & & $x$ & $x$ & $x$ & \\
\hline Bringewatt, Gershoff & 2010 & USA & CAS & & & & & & NA & NA & & $x$ & $x$ & & & & $x$ & \\
\hline Brown et al & 2014 & USA & Qual & & $x$ & & & & 20 & Urban & & $\mathrm{x}$ & & & & & & $x$ \\
\hline Cohen et al & 2012 & USA & Qual & & $\mathrm{x}$ & & & & 24 & Mixed & & $x$ & & & $x$ & $x$ & & $x$ \\
\hline Davis et al & 2012 & USA & cs & & & $x$ & & & 70 & Mixed & $x$ & $x$ & & & & & & \\
\hline Deringe et al & 2010 & USA & cs & & $x$ & & & & 1301 & Mixed & & & & $x$ & & & $x$ & $x$ \\
\hline Diamond et al & 2012 & USA & cs & & & $x$ & & & 671 & Suburban & $x$ & & & & & & & \\
\hline Draucker et al & 2005 & USA & Qual & $x$ & $x$ & $x$ & & & 63 & Urban & & & & & & & & $x$ \\
\hline Flisher et al & 1997 & USA & MM & $\mathrm{x}$ & $x$ & & & & 1285 & Urban & & & & & & & & $\mathrm{x}$ \\
\hline Galluci et al & 2005 & USA & Aud & & & & & $x$ & 5901 & NA & & & & & $x$ & & & \\
\hline Golding & 2010 & USA & CAS & & & & & & $\mathrm{NA}$ & NA & & & $x$ & $x$ & $\mathrm{x}$ & & & $x$ \\
\hline Gould et al & 2012 & USA & Aud & & & & & $x$ & $\mathrm{NA}$ & NA & & & & $\mathrm{x}$ & $\mathrm{x}$ & $x$ & $x$ & \\
\hline Hernan et al & 2010 & Australia & cS & $\mathrm{x}$ & & & & & 74 & Rural & & $x$ & $x$ & & $x$ & & $\mathrm{x}$ & $x$ \\
\hline Honingfield, McKay & 2006 & USA & CS & & & $x$ & & & 124 & Mixed & $x$ & & $x$ & & & & & \\
\hline Iskra et al & 2015 & Australia & cs & & $\mathrm{x}$ & & & & 134 & Urban & & & $x$ & $x$ & $x$ & $x$ & $x$ & \\
\hline Kerkorian et al & 2006 & USA & cs & & $\mathrm{x}$ & & & & 253 & Mixed & & & & & & & & $x$ \\
\hline McDonald et al & 2005 & Australia & Qual & & & $x$ & & & NR & NR & $x$ & $x$ & & & & & & \\
\hline McGuire, Gushard-Pine & 2005 & UK & Aud & & & & & $\mathrm{x}$ & NR & Urban & & & & & & & & $x$ \\
\hline McCann, Lubman & 2012 & Australia & Qual & $\mathrm{x}$ & & & & & 25 & Urban & & & $x$ & & $x$ & & $x$ & \\
\hline Muir et al & 2012 & Australia & Qual & $\mathrm{x}$ & & & & & 168 & Mixed & & $\mathrm{x}$ & & $\mathrm{x}$ & & & & \\
\hline Oke, Mayer & 2001 & UK & cs & & & $\mathrm{x}$ & & & 50 & Urban & $x$ & & & & & & & \\
\hline Oruche et al & 2014 & USA & Qual & $\mathrm{x}$ & $\mathrm{x}$ & & & & 24 & Urban & & & & $\mathrm{x}$ & $\mathrm{x}$ & $x$ & & $x$ \\
\hline Pfefferle & 2007 & USA & cs & & & $\mathrm{x}$ & & & 596 & Mixed & $x$ & & & & $x$ & & $x$ & \\
\hline Pullmann et al & 2010 & USA & Qual & & $\mathrm{x}$ & & $\mathrm{x}$ & & 17 & Rural & & $x$ & & & & & & \\
\hline Radovic et al & 2014 & USA & cs & & & $\mathrm{x}$ & & & 48 & Mixed & & & & & & $x$ & $x$ & \\
\hline Reid et al & 2011 & Canada & Qual & & $\mathrm{x}$ & & & & 100 & Mixed & & & & & $x$ & $\mathrm{x}$ & & \\
\hline Reid, Brown & 2008 & Canada & Qual & & & & $x$ & & 16 & Urban & $x$ & & & & $\mathrm{x}$ & $\mathrm{x}$ & & \\
\hline Richardson & 2001 & USA & cs & & $x$ & & & & 235 & Urban & & $\mathrm{x}$ & $x$ & & & $\mathrm{x}$ & & $x$ \\
\hline Sakai et al & 2014 & USA & Qual & $\mathrm{x}$ & & & & & 28 & NR & & $\mathrm{x}$ & $x$ & & & & $x$ & \\
\hline Samargia et al & 2006 & Canada & cs & $\mathrm{x}$ & & & & & 1948 & Mixed & & $x$ & $x$ & & & & $\mathrm{x}$ & $x$ \\
\hline Schraeder, Reid et al & 2014 & Canada & Qual & & $x$ & & & & 273 & Urban & & & & & $x$ & & & \\
\hline Sherman et al & 2008 & USA & Aud & & & & & $\mathrm{x}$ & 1317 & Rural & & & & & $x$ & & & \\
\hline Stern, Brown & 1994 & USA & MM & & $x$ & & & $x$ & 809 & Urban & & & & & $x$ & & & \\
\hline Stevens et al & 2006 & USA & MM & & $\mathrm{x}$ & & & $x$ & 186 & Mixed & & & & & & & & $x$ \\
\hline Stiffman et al & 2000 & USA & Qual & $x$ & & $x$ & & & 1014 & NR & & $x$ & & & & & & $x$ \\
\hline Vohra et al & 2014 & USA & cs & & $x$ & & & & 10688 & Mixed & & & & & $\mathrm{x}$ & $x$ & & $\mathrm{x}$ \\
\hline
\end{tabular}




\begin{tabular}{|c|c|c|c|c|c|c|c|c|c|c|c|c|c|}
\hline Walders et al & 2003 & USA & cs & & $x$ & & & 319 & Mixed & $x$ & $x$ & $x$ & \\
\hline Walsh et al & 2011 & UK & MM & $\mathrm{x}$ & & & & 44 & NR & & & & $\mathrm{x}$ \\
\hline Westin et al & 2012 & USA & Aud & & & & $x$ & 2054 & NR & & & $x$ & \\
\hline Williams et al & 2013 & USA & Exp & & & $x$ & & NA & Mixed & & & & \\
\hline Wisdom et al & 2006 & USA & Qual & $x$ & & & & 22 & Urban & & & & $\mathrm{x}$ \\
\hline
\end{tabular}

CYP - children, young people

RCT - randomised controlled trial

CGS - comparison group study

Cohs - cohort study

CS - cross sectional

Aud - audit

Qual - qualitative

CAS - case study

MM - mixed methods

NR - not reported

NA - not applicable 
Table 6: Finding - Improving access and engagement with CAMHS

\begin{tabular}{|c|c|c|c|c|c|c|c|c|c|c|c|c|c|c|}
\hline Authors & Year & Country & $\begin{array}{l}\text { Study } \\
\text { design }\end{array}$ & CYP & Parents & $\begin{array}{l}\text { Records/ service } \\
\text { outcomes }\end{array}$ & $\begin{array}{l}\text { MH service } \\
\text { staff }\end{array}$ & Sample size & Setting & $\begin{array}{l}\text { Providing MH services in } \\
\text { accessible location }\end{array}$ & $\begin{array}{l}\text { Facilitated } \\
\text { access }\end{array}$ & $\begin{array}{l}\text { Case assessment } \\
\text { and prioritisation }\end{array}$ & $\begin{array}{l}\text { Strategies to increase } \\
\text { engagement }\end{array}$ & CAPA \\
\hline Armbruster et al & 1997 & USA & CGS & $x$ & & & & 348 & Urban & $x$ & & & & \\
\hline Asarnow et al & 2005 & USA & RCT & $x$ & & & & 418 & NR & $x$ & & & & \\
\hline Atkins et al & 2003 & USA & MM & $x$ & $x$ & $x$ & & NR & Urban & $x$ & & & & \\
\hline Aupont et al & 2012 & USA & CohS & $\mathrm{x}$ & & & & 239 & NR & $\mathrm{x}$ & & & & \\
\hline Barwick et al & 2013 & Canada & CGS & $x$ & & & & 172 & Urban & & $x$ & & & \\
\hline Bear et al & 2014 & USA & CS & $x$ & & & & 1258 & Mixed & $x$ & & & & \\
\hline Cavaleri et al & 2009 & USA & MM & $x$ & $x$ & $x$ & & 60 CYP, 12 staff & Urban & & & & $x$ & \\
\hline Cawthorpe et al & 2007 & Canada & CS & $\mathrm{x}$ & & & & 497 & NR & & & $x$ & & \\
\hline Clark et al & 2014 & Australia & CGS & $x$ & & & & 581 & NR & & $x$ & & & \\
\hline Clemente et al & 2006 & UK & CGS & $x$ & & & $x$ & 267 & Urban & & & $x$ & $x$ & \\
\hline Dixon et al & 2011 & Australia & MM & & & $x$ & $\mathrm{x}$ & 29 & Urban & $x$ & & & & \\
\hline Eapen et al & 2012 & Australia & Aud & & & $x$ & & NR & Urban & $x$ & & & & \\
\hline Fatimilehin & 2007 & UK & MM & $x$ & $x$ & & $\mathrm{x}$ & 90 & Urban & & $x$ & & & \\
\hline Fuggle et al & 2015 & USA & MM & $x$ & & & $x$ & 54 CYP, 12 staff & Urban & & & & & $x$ \\
\hline Jones et al & 2000 & UK & Aud & & & $x$ & & 115 & Urban & & & $x$ & & \\
\hline Kaukonen et al & 2010 & Finland & CS & $x$ & & & & 949 & Mixed & & & $\mathrm{x}$ & & \\
\hline Naughton et al & 2015 & Australia & Qual & & & $x$ & & NA & Rural & & & & & $x$ \\
\hline Parker, Froese & 1992 & Australia & CGS & $x$ & & & & 385 & Urban & & & & $x$ & \\
\hline Robotham & 2009 & UK & MM & & & & $x$ & 315 & Mixed & & & & & $x$ \\
\hline Robotham et al & 2010 & UK & MM & & & & $\mathrm{x}$ & 315 & Mixed & & & & & $\mathrm{x}$ \\
\hline Smith et al & 2002 & Canada & CS & $x$ & & & $\mathrm{x}$ & 817 CYP, 92 staff & Mixed & & & $x$ & & \\
\hline Walker & 1998 & New Zealand & Aud & & & $x$ & & 143 & NR & & & & $\mathrm{x}$ & \\
\hline Wilson et al & 2015 & UK & Aud & & & $x$ & & 2896 & Mixed & & & & & $x$ \\
\hline Woodhouse & 2006 & UK & Aud & & & $x$ & & NR & Urban & & & $x$ & $x$ & \\
\hline York et al & 2004 & UK & Aud & & & $\mathrm{x}$ & & 103 & Urban & & & $x$ & & \\
\hline
\end{tabular}

CYP - children, young people

$\mathrm{RCT}$ - randomised controlled trial

CGS - comparison group study

Cohs - cohort study

CS - cross sectional

Aud - audit

Qual - qualitative

MM - mixed methods

NR - not reported

NA - not applicable 
